\title{
RESEARCH
}

\section{Monocular Clues in Seven Stereotests}

\author{
Erik Hahn, David Comstock, Stacey Durling, James MacCarron, Safiah Mulla, Paula James, Robert \\ LaRoche \\ Eye Care Team, IWK Health Centre
}

\begin{abstract}
Purpose: There have been numerous reports with evidence detailing the presence of non-stereoscopic or "monocular" clues in commonly used stereoacuity tests. The purpose of this study was to quantify the influence of monocular clues in the Titmus, Randot ${ }^{\circledR}$, Randot ${ }^{\circledR}$ Special Edition, Randot ${ }^{\circledR}$ Preschool, Lang, Lang II, and Frisby stereoacuity tests. Stereoacuity testing is typically performed and/or interpreted by eye care professionals and other health/ occupational professionals.

Methods: Two separate prospective studies were conducted. The first assessed the monocular responses of 100 subjects aged 8 to 67 with normal stereoacuity, and no previous exposure to any of the seven tests administered. The second assessed the monocular responses of 33 subjects aged 8 to 65 with longstanding, manifest, horizontal strabismus of 20 prism diopters or greater, on the aforementioned stereotests.

Results: Monocular clues were found to be present for the normal group on the Titmus (61\%), Randot ${ }^{\circledR}(6 \%)$, $\operatorname{Randot}^{\circledR}$ Special Edition (5\%), Randot ${ }^{\circledR}$ Preschool (7\%), Lang (13\%), and Lang II (37\%). Monocular clues were found to be present for the strabismic group on the Titmus (100\%), Randot ${ }^{\circledR}(9 \%)$, Randot ${ }^{\circledR}$ Special Edition (9\%), Randot ${ }^{\circledR}$ Preschool (12\%), Lang (3\%), and Lang II (27\%). There was no monocular identification for either group on the Frisby stereotest, but there was minimal binocular identification by a subject with manifest strabismus.

Conclusion: Monocular clues were present for both the normal and strabismic group on 6 of the 7 stereotests investigated. Based on these findings the authors conclude that caution must be used when interpreting patient responses on the 7 aforementioned stereotests.
\end{abstract}

\section{Introduction}

Stereopsis is the visual appreciation of depth during binocular viewing, stimulated by retinal disparity. ${ }^{1}$ Stereoacuity tests seek to determine the smallest amount of recognizable retinal disparity in seconds of arc. The measurement of stereoacuity is an important tool in the assessment of binocular function and has widely been used for the detection and management of strabismus, amblyopia and anisometropia. ${ }^{2-4}$ The response to stereoacuity testing is an important tool in the screening process for patients with suspected strabismus. Stereotests are also often used for occupational purposes to qualify individuals for a particular profession. Ideally, a stereoacuity test would be void of any monocular clues, as these would give an invalid result indicating some degree of stereopsis where none exists. There have been numerous reports in the literature examining the presence of non-stereoscopic clues in commonly used stereotests. ${ }^{3-21,24,25}$ Stereoacuity testing is typically performed by eye care professionals and other health/occupational professionals. The level of stereoacuity that cannot be obtained monocularly is of interest to all testers and interpreters of the results to provide a level of confidence that the result obtained represents stereopsis. A straightforward experimental method to determine the presence of monocular clues is to administer the test under monocular viewing conditions. We sought to quantify the presence of monocular clues in the Titmus, Randot ${ }^{\circ}$, Randot $^{\circ}$ Special Edition, Randot ${ }^{\circ}$ Preschool, Lang, Lang II, and the Frisby stereoacuity tests and determine the "absolute value" (AV) for each stereotest that could not be obtained monocularly. The AV result was not obtained by any of the subjects under monocular conditions. It is hypothesized that the AV represents a result that can only be obtained binocularly through stereopsis. We also sought to compare the monocular results of a group with normal stereopsis and a group 
with strabismus to determine if one group had superior performance under monocular conditions. We postulated that the strabismic group would have an advantage detecting monocular clues because they have adapted to utilizing monocular clues in everyday life.

\section{Material and Methods}

A total of 100 normal subjects, 60 female and 40 male, between the ages of 8 and 67 years, with a mean age of 30 years, participated in the initial study. Subjects included in the study had no previous exposure to any of the seven stereoacuity tests, and on subsequent binocular testing of stereoacuity were able to achieve 40 seconds of arc on the Titmus stereotest and at least 40 seconds of arc on the $\operatorname{Randot}^{\odot}$ stereotest. The recruited subjects reported having no known

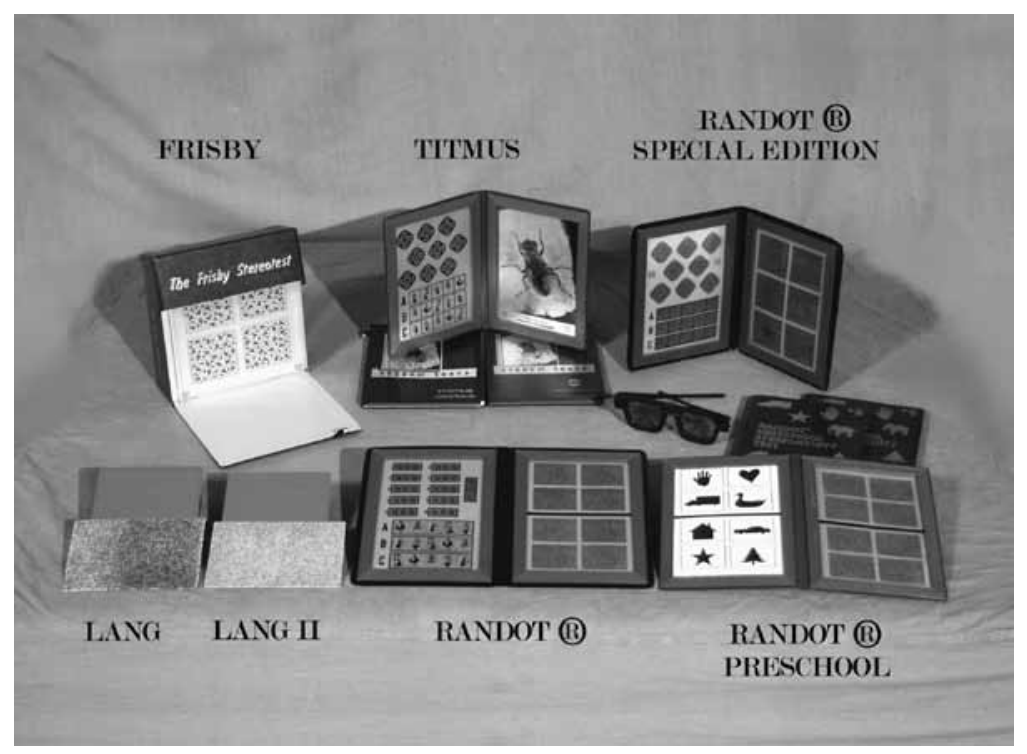

Figure 1. The seven stereotests investigated: Titmus, Randot ${ }^{\circledR}, \operatorname{Randot}^{\circledR}$ Special Edition, Randot ${ }^{\circledR}$ Preschool, Lang, Lang II, and Frisby stereoacuity tests. ophthalmologic problems other than the wearing of refractive correction (spectacles or contact lenses). The subjects were recruited by poster advertisement and word of mouth at the IWK Health Centre and Dalhousie University. The seven stereoacuity tests included were the Titmus, Randot ${ }^{\oplus}, \operatorname{Randot}^{\oplus}$ Special Edition, Randot ${ }^{\odot}$ Preschool, Lang, Lang II, and the Frisby stereoacuity tests. All tests were administered as per the manufacturers' instructions. Subjects wearing spectacle correction wore their usual correction for near tasks. Those tests requiring the use of polarized glasses were only viewed by the subjects with the polarized glasses in place (over the subject's spectacle/contact lens correction, if worn). Subjects were excluded if they did not obtain 40 seconds of arc on subsequent binocular testing. No other tests were performed on the subjects with normal stereoacuity as this level of fine stereopsis was felt to preclude any significant near visual acuity deficit. ${ }^{26}$

It is known that patients with longstanding, large angle, manifest strabismus do not demonstrate stereopsis. It is also quite evident that these individuals function very well in everyday life, presumably making use of non-stereoscopic forms of depth perception such as motion parallax, image size, linear perspective and lateral displacement. ${ }^{4}$ There has been little research conducted to determine at what level patients with longstanding, manifest, horizontal strabismus can detect monocular clues in clinical stereoacuity tests. A study by Leske and Holmes concluded that "true stereopsis" was rare in individuals with greater than 4 prism diopters of horizontal manifest strabismus. ${ }^{25}$
Therefore, for the purpose of this study, any positive response by the subjects with longstanding, manifest, horizontal strabismus of 20 prism diopters or greater was considered as a monocular clue. In order to investigate this we administered the seven stereotests to subjects with longstanding, manifest, horizontal strabismus of 20 prism diopters or greater, under both monocular and binocular viewing conditions. A positive response by any subject in this group under monocular or binocular conditions would not represent true stereopsis. After completion of the initial study on subjects with normal stereoacuity, a sample size and power calculation determined that a sample size in the range of 20 to 29 subjects with strabismus would be sufficient to detect a difference in monocular identification between the normal stereopsis and strabismic groups with $80 \%$ power and 95\% confidence. A total of 33 patients, 17 female and 16 male, aged 8 to 65 years with a mean age of 33 years, met the inclusion criteria and were included in the data analysis of the second study. All of these subjects demonstrated a longstanding horizontal strabismus of 20 prism diopters or greater (mean 38 prism diopters) at near and distance on simultaneous prism and cover test (SPCT), exhibited suppression on Worth 4-Dot testing, and had a near visual acuity of $6 / 9(20 / 30)$ or better in the fixating/dominant eye. There were 22 subjects with exotropia (mean 43 prism diopters) and 11 subjects with esotropia (mean 27 prism diopters). Subjects with dissociated vertical (or horizontal) deviation, nystagmus, or neurological disease were excluded from the study. The subjects were recruited from consenting patients at the IWK Health Centre, Eye Care Clinic. 
The Titmus Stereotest (Stereo Optical, Chicago, IL) involves a vectograph card in booklet form and a pair of polarized glasses that dissociate the eyes optically (Fig.1). Presented in the booklet are contoured stereoscopic patterns (horizontally displaced, overlapping, identical images seen separately by each eye when wearing the polarized glasses) representing a housefly (3000 seconds of arc), three rows of five animals with one animal per row imaged disparately (400 to 100 seconds of arc), and nine sets of four circles arranged in a diamond with one circle per set disparately imaged (800 to 40 seconds of arc).

Three random dot stereotests requiring the use of polarized glasses were used: $\operatorname{Randot}^{\circ}$, Randot ${ }^{\circ}$ Special Edition and Randot $^{\circ}$ Preschool (Stereo Optical). The tests are each in booklet format and present a distribution of random dots constructed using vectographic material with polarized spectacles that dissociate the eyes optically. The tasks included shape recognition, and forced choice animal and circle tasks similar to those in the Titmus stereotest.

1) The Randot ${ }^{\circ}$ combines vectographic random dot shapes on a random dot background (500 to 250 seconds of arc) and figures utilizing contoured stereoscopic patterns on a random dot background (animals 400 to 100 seconds, circles 400 to 20 seconds of arc). This test is a combination of contour and random dot targets (Fig.1). There are no criteria given in the instructions for how many correct responses on the random dot shapes constitute stereopsis.

2) The Randot ${ }^{\circ}$ Special Edition (600 to 20 seconds of arc) has only vectographic random dot figures on a random dot background. At 600 seconds there are six boxes with a shape inside each box. One shape is intentionally visible monocularly. The other five are intended to only be visible binocularly when using the polarized glasses. There are no criteria given in the instructions for how many correct responses constitute stereopsis. The number of correct responses for each subject was recorded at this level.

There are 5 shapes per row at 400, 200 and 100 seconds of arc. Again there are no criteria given in the instructions for how many correct responses constitute stereopsis. The number of correct responses for each subject was recorded at each level.

The 8 sets of circles range in disparity from 400 to 20 seconds of arc. They are arranged in a diamond pattern with four potential choices, similar to the Titmus stereotest, but are completely random dot.
3) The Randot $^{\circ}$ Preschool has 4 non-vectographic, monocularly visible shapes on the left page, and 3 vectographic images of the shapes and one blank square on the right page of 3 test booklets (800 to 40 seconds of arc). The subject "must correctly identify at least 2 of the 3 test shapes" on the right page to receive credit for that level of stereoacuity as per the manufacturer's instructions.

The Lang and Lang II stereotests (Lang, Switzerland) are each printed on a $9.5 \times 14 \mathrm{~cm}$ card and do not require the use of dissociating glasses (Fig.1). These tests utilize the principle of panography. ${ }^{22}$ Stereoscopic images of a cat, car and star in the Lang stereotest, and a car, elephant and moon on the Lang II stereotest, are embedded in random dots with separate images presented to each eye through cylindrical lenses imprinted on the surface lamination of the test card. The test card was held upright and steady by the examiner at $40 \mathrm{~cm}$ (frontoparallel) from the subject. The subject was asked "if he can see something" and then "name the objects" and "describe their distance or point to them". The cat, star and car (1200 to 550 seconds of arc) are hidden in the Lang stereotest and the elephant, car and moon (600 to 200 seconds of arc) are hidden in the Lang II stereotest. The Lang II has a monocularly visible star. The strabismic group was also asked if they could locate an area where there appeared to be an image, even if they could not identify that image. This question was added to the second study following suggestions made after presentation of the results of the first study.

The Frisby stereotest (Clement Clarke, UK) is a natural test of binocular depth perception (Fig.1). The background and target are seen in depth by being printed on opposite sides of a transparent plastic sheet (persplex $\mathrm{n}=1.49$ ). There are three plates of varying thicknesses $(6 \mathrm{~mm}, 3 \mathrm{~mm}$ and $1.5 \mathrm{~mm})$ on which triangle shaped figures are printed within four squares, one of which contains a central circle of triangles printed on the reverse side. ${ }^{9,22}$ The test plates were held by the examiner, at a distance of $30 \mathrm{~cm}$ from the patient, in front of a white background, but separated from the background to avoid shadows (600 to 150 seconds of arc at $30 \mathrm{~cm}$ ). As per the manufacturer's instructions, if the subject provided a correct response to three of the four presentations of the $6 \mathrm{~mm}$ plate they were given credit for recognizing that level.

All tests were administered as per the manufacturers' instructions with some additional questions added and noted below. 
In all testing situations, the subject's dominant eye was determined and during the initial monocular testing the non-dominant eye covered using occlusive tape. The tests were numbered 1 to 7 and presented to the subject, in a randomized order using random number tables, in the manner described. The maximum time allowed for the subject to discern any image monocularly was 15 seconds. Subsequently, the tests were presented binocularly following the same test protocol and randomized order, and the results were recorded. For all stereotests the test concluded when the subject could no longer note any "depth" to the target or the subject made two consecutive errors. Guessing was not encouraged if the subject stated that they did not perceive any depth at a particular level. The manufacturers' instructions also vary on whether a "different" response was acceptable as a correct response. For consistency, this option was offered to the subjects as a choice. The manufacturers' instructions vary on whether head and/or test movements were permitted. Again, for consistency in this study, head movements were not permitted as we were interested in monocular clues in the test design, not monocular clues that could be obtained by motion parallax, etc. The subjects were informed that head movements, or test movements, were not permitted. The examiner held the stereotest steady at the visual midline, and closed the test booklet, or removed the test from the subject's view, if they attempted to move their head during testing.

The Research Ethics Board at the IWK Health Centre approved this protocol and informed consent was obtained from each subject prior to the administration of the tests.

\section{Results}

The percentage of subjects selecting the correct response under monocular conditions was calculated. To compare differences between the subjects in the normal group and subjects in the strabismic group a t-test was used. The result was considered significant at $95 \%$ confidence $(\mathrm{p}<0.05)$. The absolute value $(A V)$ is reported as the lowest seconds of arc value that was not identified by any of the subjects in either group under monocular conditions.

The Titmus Stereotest had monocular identification up to 80 seconds of arc (Fig.2). 41\% of the subjects in the normal group and $15 \%$ of the subjects in the strabismic group were able to identify the housefly (representing 3000 seconds) as appearing in depth under monocular viewing conditions. These results were significantly different from each other $(p=0.01)$.
Monocular identification occurred for the animals representing 400, 200, and 100 seconds. At 400 seconds $3 \%$ of the normal group and $15 \%$ of the strabismic group identified the correct animal monocularly. These results were significantly different from each other $(\mathrm{p}=0.02)$. At 200 seconds 3\% of each group was able to identify the correct animal monocularly. At 100 seconds none of the normal group and 3\% of the strabismic group was able to identify the correct animal monocularly. These findings at 200 and 100 seconds were not significantly different from each other.

The circles of the Titmus test range in disparity from 800 to 40 seconds. Monocular identification occurred up to 80 seconds of arc (level 6 of 9). At 800 seconds of arc $61 \%$ of the normal group and $100 \%$ of the strabismic group identified the correct circle monocularly. These were significantly different from each other $(p=0.01)$.

At 400 seconds $36 \%$ of the normal group and 64\% of the strabismic group identified the correct circle monocularly. These were also significantly different from each other $(p=0.01)$.

At 200 seconds $6 \%$ of the normal group and 15\% of the strabismic group identified the correct circle monocularly. At 140 seconds $4 \%$ of the normal group and $9 \%$ of the strabismic group identified the correct circle monocularly. At 100 and 80 seconds $3 \%$ monocular identification occurred, only in the strabismic group. There was no significant difference between the results of the two groups from 200 to 80 seconds. There was no monocular identification at 60 , 50 or 40 seconds for either group.

The Randot $^{\oplus}$ Stereotest had monocular identification up to 140 seconds of arc (Fig.2). For the random dot shapes representing 500 and 250 seconds there was monocular identification only at 250 seconds and only in the normal group with $2 \%$ of the normal subjects correctly identifying shapes at this level. Each subject was able to identify 1 of the 3 shapes in this section.

Monocular identification occurred for the animals representing 400, 200, and 100 seconds. At 400 seconds monocular identification occurred for $5 \%$ of the normal group and $15 \%$ of the strabismic group. At 200 seconds monocular identification occurred for $3 \%$ of each group. At 100 seconds monocular identification occurred for $2 \%$ of the normal group and $3 \%$ of the strabismic group. There was no significant difference between the results of the two groups at 400, 200 or 100 seconds. 
Titmus Stereotest

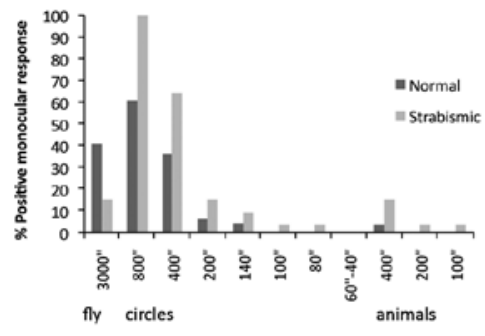

Randot Special Edition

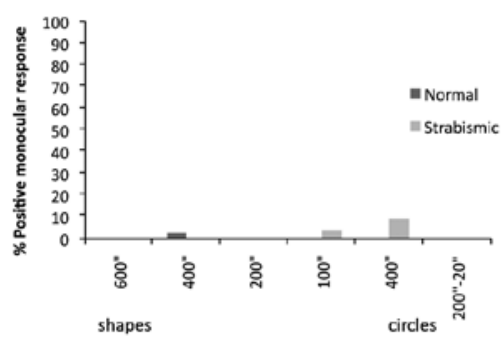

Lang Stereotest
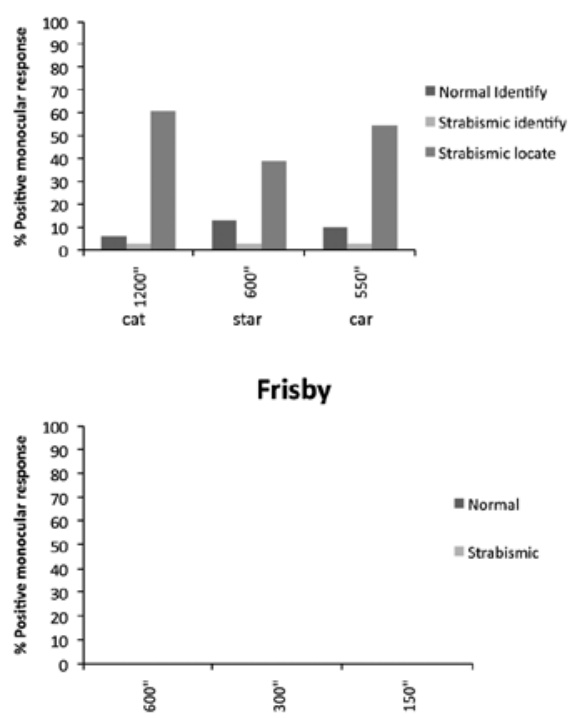

Figure 2. Monocular identification in the seven stereotests investigated.

Randot Stereotest

Lang II Stereotest

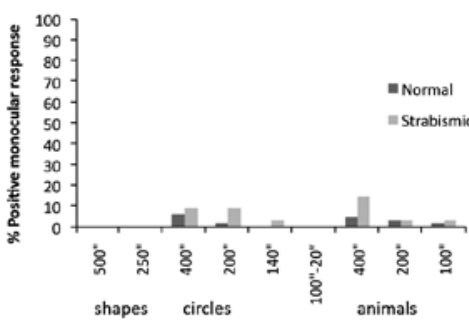

Randot Preschool Stereotest
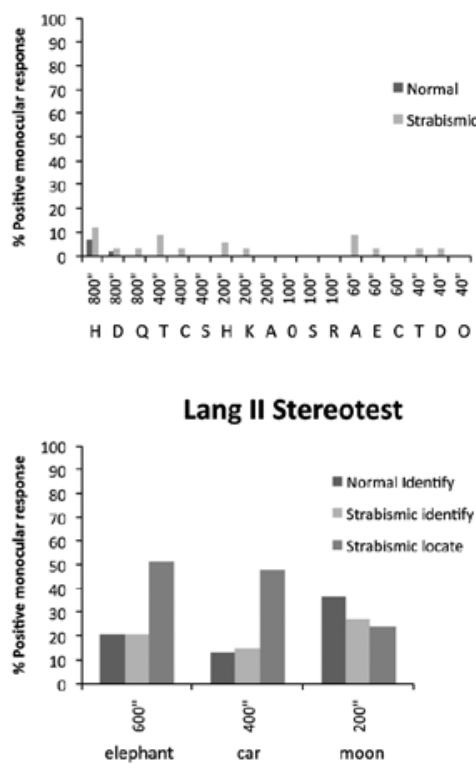

The random dot circles range from 400 to 20 seconds. Monocular identification occurred up to 400 seconds (level 1 of 8). None of the normal group was able to identify any of the circles monocularly and 9\% of the strabismic group was able to identify the 400 second circle. These were not significantly different from each other. There was no monocular identification for any of the other 7 levels of circles representing 200 to

The circles of the Randot $^{\circ}$ test range in disparity from 400 to 20 seconds. Monocular identification occurred up to 140 seconds of arc (level 3 of 10). At 400 seconds $6 \%$ of the normal group and $9 \%$ of the strabismic group identified the correct circle monocularly. At 200 seconds $2 \%$ of the normal group and $9 \%$ of the strabismic group identified the correct circle monocularly. At 140 seconds monocular identification occurred only in the strabismic group (3\%). There was no significant difference between the results of each group. There was no monocular identification for any of the other 7 levels of circles representing 100 to 20 seconds, for either group.
20 seconds, for either group.

The Randot ${ }^{\circ}$ Preschool Stereotest had monocular identification up to 40 seconds of arc (Fig.2). Only one subject in each group was able to correctly identify 2 of the 3 shapes at any level. For the normal group this identification occurred at 800 seconds. In the strabismic group one subject was able to correctly identify 2 of 3 shapes at 800,400 and 200 seconds.

The Lang Stereotest had monocular identification up to 550 seconds of arc (Fig.2). The 1200 second of arc cat was identified by $6 \%$ of the subjects in the normal group and $3 \%$ of the strabismic group. The 600 second star was identified by $13 \%$ of the normal group and $3 \%$ of 
the strabismic group. The 550 second car was identified by $10 \%$ of the normal group and $3 \%$ of the strabismic group. These results were not significantly different from each other for the two groups.

The strabismic group was additionally asked to locate areas that appeared to have an image, but which they were unable to identify. For this task $61 \%$ located the area of the 1200 second cat, $39 \%$ located the area of the 600 second star, and 55\% located the area of the 550 second car.

The Lang II Stereotest had monocular identification up to 200 seconds of arc (Fig.2). The 600 second elephant was identified by $21 \%$ of the subjects in both the normal group and the strabismic group. The 400 second car was identified by $13 \%$ of the normal group and $15 \%$ of the strabismic group. The 200 second moon was identified by $37 \%$ of the normal group and $27 \%$ of the strabismic group. These results were not significantly different from each other for the two groups.

The strabismic group was additionally asked to locate areas that appeared to have an image, but which they were unable to indentify. For the location task, 51\% were able to locate the area of the 600 second elephant, $48 \%$ located the area of the 400 second car, and $24 \%$ located the area of the 200 second moon.

The Frisby Stereotest had no monocular identification of any of the three test plates for either the normal or strabismic groups (Fig.2). There was one subject in the strabismic group that was unable to identify any of the plates monocularly, but was able to identify the 600 seconds of arc plate binocularly. This subject had alternating strabismus. These results were not significantly different from each other for the two groups.

\section{Discussion}

The participants of this study were from two distinctly different groups. The initial study had a participation criterion that included no previous exposure to any of the stereotests and a stereoacuity of 40 seconds of arc on the Titmus Stereotest and at least 40 seconds of arc on the Randot ${ }^{\circ}$, when subsequently tested binocularly. This level of stereoacuity is considered average to above average and requires a good level of binocular visual acuity. $^{26}$

The second study was comprised of subjects with long term, large angle, manifest strabismus of at least 20 prism diopters, with suppression of the non-fixating eye. Unlike the initial study, where subjects with normal
Table 1. Absolute Value (AV) of Binocular Stereoacuity. $A V$ values represent the minimum value in each stereotest that represents true stereoacuity.

\begin{tabular}{|c|c|}
\hline Stereotest & Absolute Value \\
\hline \multicolumn{2}{|l|}{ TITMUS } \\
\hline Fly & Nil \\
\hline Animals & Nil \\
\hline Circles (7/9) & $60 "$ \\
\hline \multicolumn{2}{|l|}{ Randot $^{\circledR}$} \\
\hline Shapes (any) & Nil \\
\hline Shapes $(2 / 3)$ & $500 "$ \\
\hline Animals & Nil \\
\hline Circles (4/10) & $100 "$ \\
\hline \multicolumn{2}{|l|}{ Randot $^{\circledR}$ Special Edition } \\
\hline Shapes 600" any & Nil \\
\hline Shapes $600 "(3 / 5)$ & Nil \\
\hline Shapes 600" (4/5) & $600 "$ \\
\hline Shapes 400-100" any & Nil \\
\hline Shapes 400-100" (3/5) & $400 "$ \\
\hline Circles (2/8) & $200 "$ \\
\hline \multicolumn{2}{|l|}{ Randot ${ }^{\circledR}$ Preschool } \\
\hline Shapes $(2 / 3)$ & $100 "$ \\
\hline Lang & Nil \\
\hline Lang II & Nil \\
\hline Frisby & $300 "$ \\
\hline
\end{tabular}

stereoacuity were made artificially acutely monocular and asked to identify monocular clues, these subjects have adapted to a life that is dependent on monocular clues. Our question was whether these subjects could perform better than the normal group and exhibit higher monocular, falsely positive, stereoacuity levels. On the Titmus, Randot ${ }^{\circ}$, Randot ${ }^{\circ}$ Special Edition, Randot ${ }^{\circ}$ Preschool, Lang, and Frisby stereoacuity tests the strabismic group preformed better and lowered the AV for each test. These differences were not statistically significant at the lower stereoacuity levels, but in the calculation of an AV these differences require clinical consideration. The purpose of this study was to determine the AV that was not attained monocularly by any of the subjects in either group (or binocularly in the strabismic group). 
Stereotests are designed to detect the presence of stereopsis and quantify the level of stereoacuity. Clinicians desire to have assurance that the stereoacuity measured is the result of binocular depth perception, and not a measure of disparity that can be detected monocularly. Therefore it is presumed that, in this study population, the AV can only be attained through stereopsis.

The Titmus Stereotest is probably the most widely recognized and used stereotest in North America. It has always been manufactured by Stereo Optical (Chicago, IL) and was brand labeled for the Titmus Optical Company. It is now only available from Stereo Optical (SO-001), and is known as the "Stereo Fly." There have been many comments in the literature suggesting the presence of monocular clues in the Titmus Stereotest. 1,4, 5-7,9,14-17,19,25

The "housefly" image in this test has one of the greatest commercially available disparities at 3000 seconds of arc. $41 \%$ of the subjects in the normal group identified the fly as appearing in depth when they viewed it monocularly through the polarized spectacles. Only $15 \%$ of the subjects in the strabismic group claimed to appreciate depth during monocular viewing. This significant difference $(\mathrm{p}=0.01)$ was surprising when one would expect if the "depth" of the fly was from monocular clues, then the strabismic group would have a greater percentage claim that the image appears in depth. The difference could lie in the presentation of a large, glossy, two dimensional image to an acutely monocular group, giving the image the appearance of depth. The subjects in the experienced monocular group (the subjects with strabismus) may have a greater knowledge that two dimensional objects are flat, and/ or likely had previous exposure to the "fly," and know the correct answer for them is "flat."

The animal section of the test represents 400, 200, and 100 seconds. The combined results indicate that this section could not be used for occupational and other such purposes (AV - nil).

The circle test had slightly variable results comparing the normal and the strabismic group. For the normal group the AV was 100 seconds. For the strabismic group the AV was 60 seconds of arc. Interestingly, the strabismic group performed significantly better at monocular identification $(\mathrm{p}=0.01)$ for both the 800 and 400 seconds of arc circle. The results of these groups combined leave the clinician with an AV of 60 seconds (7/9 circles) (Table.1). This is slightly lower than previously reported levels where 100 seconds was considered as a positive response. ${ }^{16,25}$

With the exception of the fly, the strabismic group had greater monocular perception on both the circles and the animals. Possible explanations for this result could rest in the fact that the normal group contained subjects that had not received any previous exposure to the stereotests used in this investigation. The strabismic group, in all probability, had multiple previous exposures to this stereotest, given its popularity. It is also possible that the normal group, made acutely monocular, was not as skilled at noting monocular clues as the strabismic group. To determine if the strabismic group is truly better at detecting monocular clues, a completely novel stereotesting situation would need to be created.

The Randot $^{\circ}$ Stereotest (SO-002) is an unusual combination of figures. One page of the test is random dot while the other page is contoured stereoscopic patterns on a random dot background. The random dot shapes represent 500 and 250 seconds of arc. The clinician cannot be certain of an AV for occupational, purposes, etc., unless requiring the subject to identify at least two of the three shapes. The manufacturer's instructions do not provide a number of correct responses for this section to be considered accurate. Using 2 of the 3 shapes present as criterion for successful identification, the AV is 500 seconds for the shapes section.

The animal section of this test is very similar to that used in the Titmus Stereotest. The animals are the mirror image of the Titmus animal section and are not random dot figures. They are contoured stereoscopic patterns superimposed on a random dot background. The AV for this section was nil for both the normal and strabismic group as there was monocular identification at all levels. This result was the same for the Titmus which also did not have an AV for the animals as there was monocular identification at all levels of animals on that stereotest as well. It appears that although the animals are the mirror image of the Titmus test, the random dot background does not make it more difficult to identify the animals monocularly. These combined results indicate that this section should not be used for occupational and other such purposes (AV - nil).

The circle section of the Randot ${ }^{\circ}$ stereotest is arranged differently than that in the Titmus. It is composed of ten sets of three circles that are linearly arranged. Again, it is not a true random dot stereotest; rather it utilizes contoured stereoscopic patterns on a random 
dot background. The AV for the combined group was 100 seconds. This compares to the AV of the Titmus test at 60 seconds. It appears that even though there are only three choices in the Randot ${ }^{\circ}$ circle test, the linear arrangement on a random dot background is more difficult to identify monocularly than the diamond arrangement, on a black background, of the nine sets of four circles in the Titmus Stereotest. Therefore clinically we can accept an AV of 100 seconds (4/10) (see Table 1). This is lower than previously reported levels where 160 seconds was considered as a positive response. ${ }^{27}$

The Randot ${ }^{\circ}$ Special Edition had excellent results. The manufacturer's instructions do not provide a number of correct responses for the stereoscopic shapes at the 600 seconds of arc level to be considered accurate. This section has an AV of 600 seconds, if 4 out of 5 are the accepted criteria.

The random dot shapes representing 400, 200, and 100 seconds respectively had minor amounts of monocular identification. Again, the manufacturer's instructions do not provide a number of correct responses for this section to be considered accurate. This section has an $\mathrm{AV}$ of 400 seconds, if 3 out of 5 are the accepted criteria.

The random dot circles represent a stereoscopic range from 400 to 20 seconds. The style is similar to the Titmus circles, but the test is completely random dot. The normal group had no monocular identification of the circles, while $9 \%$ of the strabismic group was able to identify the 400 second circle. The AV for the circle test is 200 seconds (2/8). This is a higher threshold than either of the circle sections of the Titmus or Randot ${ }^{\circ}$ stereotests (see Table 1).

Cooper et al reported that none of their subjects responded at better than chance probability level when the Randot ${ }^{\circ}$ Special Edition Circle test was given monocularly. ${ }^{11}$ Our data also demonstrate excellent results in this section of the test as any measurement at 200 seconds ( 2 or more of the 8 sets of circles) can be considered a true measure of stereoacuity. Despite the excellent results found in this and other investigations, as the name implies, this test is only available as a special group order from Stereo Optical and is known as the Original Randot (SO-006). It can be obtained individually from Bernell VTP and is listed as the Paul Harris Randot Test (Special Edition) Item \# SORDTPH.

The Randot $^{\ominus}$ Preschool Stereotest (SO-007) has matching pictures on the left page for random dot shapes on the right page. There are four pictures on the left page but only 3 of the 4 appear on the random dot side. The manufacturer suggests that a subject should only be given credit for a level of stereoacuity if the subject correctly identifies 2 of the 3 images correctly.

There were several subjects in each group that were able to identify 1 of the 3 pictures at various levels of stereoacuity (Fig.2). Only one subject in each group was able to identify 2 of the 3 images. In the normal group this identification occurred only at 800 seconds of arc while in the strabismic group the identification occurred at 800,400 and 200 seconds. Therefore, using 2 out of 3 correct responses as the criteria, the AV is 100 seconds (Table 1). This is lower than the results of Leske and Holmes where 400 seconds was considered a positive response, and similar to the results of Fawcett and Birch who suggested examiner confidence at 160 seconds or better. ${ }^{25,27}$

Our tested population was older than the intended "preschool" population that this test series was designed to target. A preschool population may have different results than those of our tested population. It is possible that any response in the preschool age range of 2 out of 3 may represent clinical significance, but this has yet to be determined. The manufacturer's instructions do not provide any information on how the blank space should be scored. It can be assumed that it should be ignored as the successful score indicated by the manufacturer's instructions is 2 out of 3 , and there are only 3 pictures on the random dot side. The 4 picture shapes to match with 3 random dot figures may lead to guessing if the subject assumes there are 4 choices and guesses the blank space as the fourth picture. This could be confusing for the subjects.

Further study of the Randot ${ }^{\circ}$ Preschool Stereotest could be performed on a preschool population and consider the probability of the blank space. It has been reported that the test itself has also been modified and the heart shape has been changed. ${ }^{25}$ Our testing was performed with the original test in three separate booklet forms. Our results may not apply to those using the revised version of the test.

The range of disparity in the Lang Stereotest is 1200 to 550 seconds of arc. The Lang II Stereotest has a range of disparity from 600 to 200 seconds. The Lang Stereotest had less monocular identification of objects than the Lang II Stereotest. In the second part of this study, the subjects in the strabismic group were also asked to locate areas that appear to have an image, but which they were unable to identify. Many of these subjects were able to identify the correct location 
of these images, even though they were unable to identify the object correctly. The authors are aware of anecdotal suggestions that correct localization of the areas where an image is located could be considered true stereoacuity. After completion of the initial study it was suggested that we examine localization without identification in the strabismic group. Lang has suggested that location of the area of an image, without recognition, requires further examination. ${ }^{22}$ Our findings indicate that Lang's suggestion should be adopted. The subjects in the strabismic group had a much lower identification than localization of the Lang shapes.

Our results indicate that the Lang results have to be interpreted with caution. Localization of a distorted area should not be considered as a positive response, and even identification of objects should be regarded with caution. The original Lang Stereotest has less monocular identification than the Lang II, which has a lower disparity in seconds of arc and should, in theory, be more difficult. From these results there can be no $\mathrm{AV}$ because there was monocular identification of all figures (Table 1).

There were no subjects in either the normal or strabismic groups that could successfully identify the target in the Frisby Stereotest under monocular conditions. Our results are identical to those of Holmes and Leske who tested 100 monocularly occluded normal adults and found that none of their subjects were able to pass the test. ${ }^{21}$ However, in the strabismic group of our investigation, there was one individual who was able to successfully identify the 600 seconds of arc plate binocularly. This individual was noted to have alternating exotropia of a large magnitude (AXT 65 a ) and may have benefitted from an alternate fixation strategy described by Archer. ${ }^{24}$ Success on the Frisby Stereotest in an individual with alternating strabismus has also been reported by Hall. ${ }^{14}$ In a study of stereoacuity in individuals with strabismus, Leske and Holmes did not encounter any subjects that were able to successfully identify the Frisby target using an alternate fixation strategy. ${ }^{25}$

Our results lead us to recommend an $\mathrm{AV}$ of 300 seconds (see Table 1). The AV could be expanded to 600 seconds if individuals with alternating strabismus are able to be detected and excluded. It appears that individuals able to identify 600 seconds binocularly via alternating strabismus are rare. This was the only result in which a subject with strabismus performed better binocularly than monocularly. However, the purpose of this study was to identify a level that could not be attained falsely by any of the subjects. A subject in the strabismic group should not have any stereopsis. Although the positive response of the subject at 600 seconds of arc was not due to monocular clues it prevents us from recording 600 as the AV as this response did not represent true stereoacuity. An AV of 300 seconds still ranks the Frisby Stereotest as the highest binocular AV of the stereotests investigated in this study. It is a very easy to use test and, as shown, has no monocular clues and can only be perceived binocularly by individuals with stereoacuity and rarely by individuals with alternating strabismus at 600 seconds of arc.

Overall, our results of AVs are summarized in Table 1. This table is useful as a clinical reference when interpreting the results of stereoacuity testing. The clinician can quickly check the table for confidence in the level obtained/reported as an AV representing true stereopsis.

As clinicians we do not wish to discount the importance of astute clinical judgment of the presence of peripheral stereoacuity, which is beyond the AVs determined in this study. The authors have all seen a child pass a hand through the perceived space of the elevated wings of the Titmus Fly, yet not be able to identify any of the targets beyond that level. This surely represents stereoacuity, even though it has been shown in this and other studies that many of the targets of the Titmus stereotest can be identified monocularly. The 3000 second level has certainly proven useful in our clinic with many of these individuals, and/or their parents, who report experiencing, or observing their child also responding to, the "depth effect" of 3-D movies. It is this high level of disparity that helps the Titmus/Stereo Fly stereotest retain its popularity despite numerous reports of monocular clues. Tests such as the Frisby Stereotest and the Randot ${ }^{\circ}$ Special Edition Stereotest have better AV levels yet remain less popular. This may lie in the fact that the most peripheral level of these tests is 600 seconds compared to the 3000 second level of the Titmus/Stereo Optical Fly. As clinicians we are confident in our clinical ability to judge a positive response in young children to a variety of stereotests. A positive response to a battery of tests increases our clinical confidence. However, we have also seen infants as young as 6 months reach out to grasp the "circle of triangles" of the Frisby Stereotest at 600 seconds and have confidence that stereoacuity exists even though they are too young to perform any of the other tests.

In conclusion, we advocate astute clinical judgment for measured stereoacuity that is more peripheral than the AV levels reported in this study. The AV levels are useful 
for occupational stereoacuity requirements, especially when the administrator of the test may not be an eye care professional, or the professional interpreting the result was not present for the testing and/or does not have any knowledge of the individual's ophthalmologic status. For example, the Canadian Armed Forces requires a stereoacuity level of 40 seconds of arc on the Titmus Stereotest for recruits wishing to enter pilot training. This level appears to be a safe judgment of true stereoacuity, from the results of this and other studies, which is unlikely to be affected by the expertise of the test administrator (see Table 1).

For mature subjects, we advocate the use of traditional clinical methods of confirming suspected stereoacuity results by eliminating the disparity (covering the non-dominant eye, turning the test 90 degrees) or reversing the disparity (turning the test 180 degrees for tests with polarized glasses, or reversing the front side of the plate in the Frisby) and asking the subject to report on the apparent change. For occupational purposes, the AV levels could be employed where true stereopsis is deemed to be an occupational requirement.

\section{Acknowledgments}

The authors would like to acknowledge Colleen O'Connell Ph.D. Dalhousie University, IWK Health Centre, Research Associates, Perinatal Epidemiology Research Unit, for her statistical assistance.

\section{References}

1. Mazcow ML, Prager TC, Cathy GC. Assessment of three stereoacuity tests. American Orthoptic Journal 1983;33:111-115.

2. Birch EE, Williams C, Hunter J, Lapa MC, ALSPAC Children in Focus Study Team. Random dot stereoacuity in preschool children. J Pediatr Ophthalmol Strabismus 1997;34:217-22.

3. Fricke T, Siderov J. Non-stereoscopic clues in the Random-dot E stereotest: Results for adult observers. Ophthal and Physiol Opt 1990;10:133-136.

4. Von Noorden GK, Campos EC. Binocular vision and ocular motility: Theory and management of strabismus 6th ed. Toronto: Mosby; 2002:21-28,298-307.

5. Levy NS, Slick EB. Stereoscopic perception and Snellen visual acuity. Am J Ophthalmol 1974;78: 722-724.

6. Reineck RD, Simons K. A new stereoscopic test for amblyopia screening. Am J Ophthalmol 1974;78:714-721.

7. Simons K, Reinecke RD. A reconsideration of amblyopia screening and stereopsis. Am J Ophthalmol 1974;78:707-13.

8. Cooper J, Warshowsky J. Lateral displacement as a response cue in the Titmus stereo test. Am J Optom Physiol Optics 1977;54:537541.

9. Hinchcliffe HA. Clinical evaluation of stereopsis. Br Orthopt J 1978;35:46-57.

10. Cooper J, Feldman J. Assessing the Frisby stereo test under monocular viewing conditions. J Am Optometric Assoc 1979;50:807-809.

11. Cooper J, Feldman J, Medlin D. Comparing stereoscopic performance of children using the Titmus, TNO, and Randot stereo tests. J Am Optom Assoc 1979; 50:821-825.
12. Simons K. Stereoacuity norms in young children. Arch Ophthalmol 1981;99:439-445.

13. Simons K. A comparison of the Frisby, Random - Dot E, TNO, and Randot circles stereotests in screening and office use. Arch Ophthalmol 1981;99:446-452.

14. Hall C. The relationship between clinical stereotests. Ophthal Physiol Opt 1982; 2:135-143.

15. Rutstein RP, Eskridge B. Stereopsis in small-angle strabismus. Am J Optom Physiol Opt 1984;61:491-498.

16. Broadbent $\mathrm{H}$, Westall $\mathrm{C}$. An evaluation of techniques for measuring stereopsis in infants and young children. Ophthal and Physiol Opt 1990;10:133-136.

17. Clarke WN, Noel LP. Stereoacuity testing in monofixation syndrome. J Pediatr Ophthalmol Strabismus 1990;27:161-163.

18. Manny RE, Martinez AT, Fern KD. Testing stereopsis in the preschool child: Is it clinically useful? J Pediatr Ophthalmol Strabismus 1991;28:223-231.

19. Schiveers MA, Baker JD. Comparison of Titmus and two Randot tests in monofixation. Am Orthoptic J 1992;42:135-142.

20. Fricke T, Siderov J. Non-stereoscopic clues in the Random-dot E stereotest: Results for adult observers. Ophthal Physiol Opt 1997;17:122-127.

21. Holmes JM, Leske DA. Monocular clues in tests of stereoacuity. In: Pritchard C., editor. Transactions IX International Orthoptic Congress. Nurmberg, Germany: Berufsverband der Orthoptistinner Deutchlands eV; 1999;103-6.

22. Lang, J. The two-pencil test and the new Lang Stereotest. Brit Orthopt J 1984;41:15-21.

23. Saunders, KJ, Woodhouse MJ, Westall CA. The modified Frisby stereotest. J Pediatr Ophthalmol Strabismus 1996;33:323-327.

24. Archer SM. Stereotest artifacts and the strabismus patient. Graefes Arch Clin Exp Ophthalmol. 1988;226:313-6.

25. Leske DA, Holmes JM. Maximum angle of horizontal strabismus consistent with true stereopsis. J AAPOS 2004;8:28-34.

26. Odell NV, Hatt SR, Leske DA, Adams WE, Holmes JM. The effect of induced monocular blur on measures of stereoacuity. J AAPOS 2009;13(2):136-141.

27. Fawcett SL, Birch EE. Validity of the Titmus and Randot circles in children with known binocular vision disorders. J AAPOS 2003;7(5):333-338. 